Preface

\title{
Fibrinolysis: Biochemistry, Clinical Aspects, and Therapeutic Potential
}

\author{
Hau Kwaan, MD ${ }^{1}$ Ton Lisman, PhD ${ }^{2}$ Robert L. Medcalf, PhD ${ }^{3}$ \\ ${ }^{1}$ Division of Hematology/Oncology, Feinberg School of Medicine, \\ Northwestern University, Chicago, Illinois \\ ${ }^{2}$ Section of Hepatobiliary Surgery and Liver Transplantation, \\ Department of Surgery, University Medical Center Groningen, \\ University of Groningen, Groningen, The Netherlands \\ ${ }^{3}$ Molecular Neurotrauma and Haemostasis, Australian Centre for \\ Blood Diseases, Monash University, Clayton, Australia
}

Semin Thromb Hemost 2017;43:113-114.

The fibrinolytic system is responsible for physiological lysis of hemostatic plugs once vascular integrity has been restored after injury. The formation and regulation of plasmin proceeds in a very similar way to the formation and regulation of thrombin in the coagulation cascade. ${ }^{1}$ Complex regulatory pathways, which include cell surfaces, the fibrin surface, the structure of the fibrin clot, and circulating inhibitors regulate physiological clot lysis. The physiological relevance of the fibrinolytic system is evidenced by rare bleeding disorders due to accelerated fibrinolysis such as Quebec platelet disorder, congenital deficiencies of antiplasmin and plasminogen activator inhibitor type 1 (PAI-1), and acquired hyperfibrinolytic states such as observed in acute promyelocytic leukemia. ${ }^{2}$ In addition, efficacy of exogenous administration of plasminogen activators to lyse pathological thrombi underlines the therapeutic importance of the fibrinolytic system. Further to the physiological role of fibrinolysis in intravascular clot lysis, components of the fibrinolytic system play an important role in many physiological and pathological processes unrelated to intravascular clot lysis. $^{3}$

In this issue of Seminars of Thrombosis \& Hemostasis, we have assembled articles dealing with new developments in biochemistry, clinical aspects, and the therapeutic potential of the fibrinolytic system. This collection of articles describes new developments and covers topics not included in a recent issue of the journal in 2013. ${ }^{4}$

Although primary, secondary, and tertiary hemostases were traditionally viewed as separate processes, each acting on a different timescale, we currently understand that all components of hemostasis act simultaneously and have complex interactions. The first part of this issue discusses new insights into the biochemistry of fibrinolysis. In the first article, by Whyte et al, the role of platelets in clot stability and lysis is outlined. ${ }^{5}$ The authors discuss recent insights in platelet-mediated modulation of fibrinolysis by secretion of fibrinolytic proteins from platelet granules and by plateletsurface modulation of fibrinolysis. Importantly, platelets appear to have both pro- and antifibrinolytic properties, the net effect of which is incompletely understood. The next article by Vallier et al describes recent observations on the profibrinolytic properties of microparticles shed from various cell types. ${ }^{6}$ In addition to the well-known procoagulant properties of microparticles, there is accumulating data showing that microparticles can initiate plasmin generation. The potential role of fibrinolytic microparticles in detection and pathogenesis of cancer is also discussed.

The next part of this issue deals with functions of the fibrinolytic system beyond lysis of hemostatic plugs or pathological thrombi. The article by Foley discusses the role of the fibrinolytic system in inflammation and as a regulator of the complement system. ${ }^{7}$ Specifically, the article deals with the roles of fibrinolytic components in infection, wound healing, and inflammation and includes diseases such as atherosclerosis. Draxler et al then further describe the role of plasmin as a modulator of the immune response and specifically address the role of plasmin in tissue homeostasis and the detrimental effects of dysregulated plasmin generation in chronic and acute inflammatory states. ${ }^{8}$ The possibility that antifibrinolytic agents act also as antiinflammatory drugs is discussed. This additional function of antifibrinolytic agents may be relevant for patients with trauma or undergoing major surgery that receive antifibrinolytic drugs. The next article by Fredriksson et al deals with nonfibrinolytic effects of tissue plasminogen activator (tPA) in the central nervous system. ${ }^{9}$ Some controversy on the
Address for correspondence Hau C. Kwaan, MD, FRCP, Divisio of Hematology/Oncology, Feinberg School of Medicine, Northwestern University, Chicago, IL 60611 (e-mail: h-kwaan@northwestern. edu).
Issue Theme Fibrinolysis: Biochemistry, Clinical Aspects, and Therapeutic Potential; Guest Editors: Hau Kwaan, MD, Ton Lisman, PhD, and Robert L. Medcalf, PhD,
Copyright $\odot 2017$ by Thieme Medical Publishers, Inc., 333 Seventh Avenue, New York, NY 10001, USA.

Tel: +1(212) 584-4662.
DOI http://dx.doi.org/ 10.1055/s-0036-1598000. ISSN 0094-6176. 
effects of tPA on physiology and pathophysiology within the central nervous system remains unresolved. Specifically, both neuroprotective and neurotoxic effects of tPA have been described. The authors propose a unifying theory for these pleiotropic effects of tPA within the central nervous system. One other function of the fibrinolytic system beyond intravascular clot lysis discussed in this issue is its role in fibrosis. The subsequent article by Flevaris and Vaughan discusses the profibrotic properties of PAI-1 in liver, lung, and kidney, which are mediated by inhibition of plasminmediated activation of matrix metalloproteases, and the incompletely characterized antifibrotic properties of PAI-1 in the heart. ${ }^{10}$

The final part of this issue deals with clinical aspects of fibrinolysis. First, Lisman addresses the link between a hypofibrinolytic state, as assessed with a plasma-based clot lysis assay, and the risk for venous and arterial thromboses in the general population. ${ }^{11}$ Next, Campbell addresses the clinical management of ischemic stroke, where recent developments in using thrombectomy in combination with thrombolytic therapy have shown favorable results compared with thrombolytic therapy alone in five clinical trials performed in $2015 .^{12}$ The next article by Reed et al discusses the role of antiplasmin in ischemic stroke. ${ }^{13}$ Specifically, epidemiological evidence and studies in experimental animals indicate that high levels of antiplasmin increase the risk for stroke and its complications. Pharmacological modulation of antiplasmin levels decreases stroke severity in animal models, and may provide an additional new therapeutic approach in the management of ischemic stroke. Subsequently, Walsh et al discuss changes in the fibrinolytic system in patients with trauma and address the evidence for the existence of hyper- and hypofibrinolytic conditions in these patients. ${ }^{14}$ These findings are put into clinical perspective in two subsequent articles discussing the use of tranexamic acid in trauma patients. First, Walsh et al discuss the perspective from the United States in which the use of tranexamic acid in trauma is treated with caution. ${ }^{15}$ Gall et al subsequently discuss the current European policy, which promotes much more liberal use of this antifibrinolytic agent in all bleeding trauma patients (both suspected and confirmed), those that require immediate blood transfusion, and all severely injured patients with evidence of hemorrhagic shock receive antifibrinolytic therapy as early as possible. ${ }^{16}$ Debate using arguments in favor and against a liberal use of tranexamic acid in trauma will hopefully lead to a continuing dialog and design of clinical studies aimed at finding the optimal use of antifibrinolytic drugs for this indication.

In summary, this issue of Seminars in Thrombosis and Hemostasis highlights the many facets of fibrinolysis in health and disease and accentuates the continuing need for clinical development of both pro- and antifibrinolytic strategies. We hope you enjoy the variety of topics discussed herein.

\section{References}

1 Chapin JC, Hajjar KA. Fibrinolysis and the control of blood coagulation. Blood Rev 2015;29(1):17-24

2 Kolev K, Longstaff C. Bleeding related to disturbed fibrinolysis. Br J Haematol 2016;175(1):12-23

3 Draxler DF, Medcalf RL. The fibrinolytic system-more than fibrinolysis? Transfus Med Rev 2015;29(2):102-109

4 Kwaan HC, Mazar AP. Biologic role of the plasminogen-plasmin system: thrombolysis, bleeding, and beyond. Semin Thromb Hemost 2013;39(4):327-328

5 Whyte CS, Mitchell JL, Mutch NJ. Platelet-mediated modulation of fibrinolysis. Semin Thromb Hemost 2017;43(2):115-128

6 Vallier L, Cointe S, Lacroix R, et al. Microparticles and fibrinolysis. Semin Thromb Haemost 2017;43(2):129-134

7 Foley JH. Plasmin(ogen) at the nexus of fibrinolysis, inflammation and complement. Semin Thromb Haemost 2017;43(2):135-142

8 Draxler DF, Sashindranath M, Medcalf RL. Plasmin: a modulator of immune function. Semin Thromb Haemost 2017;43(2):143-153

9 Fredriksson L, Lawrence DA, Medcalf RL. tPA modulation of the blood-brain barrier: a unifying explanation for the pleiotropic effects of tPA in the CNS? Semin Thromb Haemost 2017;43(2): 154-168

10 Flevaris P, Vaughan D. The role of plasminogen activator inhibitor type-1 in fibrosis. Semin Thromb Haemost 2017;43(2):169-177

11 Lisman T. Decreased plasma fibrinolytic potential as a risk for venous and arterial thrombosis. Semin Thromb Hemost 2017; 43(2):178-184

12 Campbell BCV. Thrombolysis and thrombectomy for acute ischemic stroke: strengths and synergies. Semin Thromb Hemost 2017;43(2):185-190

13 Reed GL, Houng AK, Singh S, Wang D. $\alpha_{2}$-Antiplasmin: new insights and opportunities for ischemic stroke. . Semin Thromb Hemost 2017;43(2):191-199

14 Walsh M, Shreve J, Thomas S, et al. Fibrinolysis in trauma: "Myth", "Reality" or "Something in Between". Semin Thromb Haemost 2017;43(2):200-212

15 Walsh M, Thomas S, Moore E, et al. Tranexamic acid for trauma resuscitation in the United States of America. Semin Thromb Haemost 2017;43(2):213-223

16 Gall LS, Brohi K, Davenport RA. Diagnosis and treatment of hyperfibrinolysis in trauma: (A European perspective). Semin Thromb Haemost 2017;43(2):224-234 